\title{
Über das Vorkommen von Cholesterinestern im Gehirn.
}

Von

Dr. R. Bünz.

(Aus der chemischen Abteilung des physiologischen Instituts zu Berlin.)

(Der Redaktion zugegangen am 5. August 1905.)

Von Baumstark ${ }^{1}$ ) wurde die Angabe gemacht, daß das Gehirn nicht nur Cholesterin, sondern auch gebundenes Cholesterin - vielleicht als Ölsäureester - enthält und daß letzteres sogar an Menge ersteres übertrifft. Diese Angabe ist in die Lehrbücher übergegangen.

Bei genauerer Prüfung der Experimente jedoch, auf Grund derer Baumstark zu diesem Resultat gekommen ist, ergibt sich, daß der Beweis für die Existenz derartig gebundenen Cholesterins keineswegs erbracht ist. Baumstark benutzte zur Entfernung des Cholesterins aus den ätherischen Gehirnauszügen die Eigenschaft dieser Substanz, sich in warmem Alkohol zu lösen, um beim Erkalten wieder auszukristallisieren. Er behandelt daher das ätherische Extrakt so lange und so oft mit Alkohol bei $40-50^{\circ}$, als die abgegossenen alkoholischen Lösungen beim Erkalten noch Cholesterin abscheiden. Die vereinigten alkoholischen Mutterlaugen engt er bei $40^{\circ}$ auf ein kleines Volumen ein. Den dabei resultierenden Rückstand, welcher eine mit Cholesterinkristallen durchsetzte, rötlichgelbe, ölig schmierige Masse darstellt, rührt er mit wenig $85 \%$ igem Alkohol bei $40^{\circ}$ an, wobei sich nur das Öl löst, filtriert über Glaswolle von den ungelösten Cholesterinkristallen ab und verdunstet bei $40^{\circ}$. Diese Operation wiederholt er so lange, bis sich auch beim Abkühlen unter $0^{\circ}$ keine Spur Cholesterin aus der alkoholischen Lösung abscheidet.

1) Diese Zeitschrift, Bd. IX, S. 163. 
Auf diese Weise glaubte Baumstark einen in Alkohol leicht löslichen, von Cholesterin vollkommen befreiten Gehirnauszug erhalten zu haben. Als er nun nach der Verseifung mit alkoholischer Kalilauge wiederum reichliche Mengen Cholesterin aus dieser Masse erhielt, meinte er, aus diesem Befunde auf das Vorhandensein von gebundenem Cholesterin schließen zu dürfen, und zwar vermutet er wegen der dabei auftretenden Ölsäure einen Cholesterinester dieser Säure.

Es liegt auf der Hand, daß hier ein falscher Schluß vorliegt. Die in reichlicher Menge in der Ätherlösung vorhandenen Stoffe, wie Lecithin, vermögen bedeutende Quantitäten Cholesterin in Lösung $\mathrm{zu}$ halten, welche sich durch das von Baumstark benutzte Verfahren mittels Alkohol nicht nachweisen lassen, wohl aber nach der Behandlung mit alkoholischer Kalilauge.

Da wir nun in dem Aceton ein Mittel kennen gelernt haben, mit Hilfe dessen Cholesterin in sehr viel vollkommenerer Weise, als es bisher möglich war, vom Lecithin und ähnlichen Stoffen zu trennen ist (G. Zuelzer), ${ }^{1}$ ) so veranlaßte mich Herr Prof. Thierfelder, die Angaben von Baumstark nachzuprüfen.

Über die Versuche soll im folgenden kurz berichtet werden:

Etwa $650 \mathrm{~g}$ von Blut und Häuten befreites und zerkleinertes, frisches Pferdegehirn wurde mit der anderthalbfachen Menge entwässerten Natriumsulfats verrieben und darauf durch ein feines Sieb getrieben. Auf diese Weise erhielt ich ein vollkommen trockenes und feines, für die weitere Bearbeitung außerordentlich geeignetes Pulver. Dieses wurde so oft mit Äther ausgeschüttelt, bis im Verdunstungsrückstande einer Probe des abgegossenen Äthers kein Cholesterin mehr nachzuweisen war. Die Ätherauszüge wurden vereinigt, eingeengt und mit dem doppelten Volumen Aceton versetzt. Nachdem der entstandene Niederschlag sich abgesetzt hatte, wurde die Flüssigkeit abfiltriert, der Niederschlag wieder in Äther gelöst, die ätherische Lösung abermals mit Aceton gefällt und das Verfahren in gleicher Weise noch zweimal wiederholt. Ich erhielt so einen in Aceton unlöslichen Rückstand und vier AcetonÄtherlösungen.

') Diese Zeitschrift, Bd. XXVII, S. 265. 
Der Rückstand wurde in Alkohol gelöst und mit alkoholischer Kalilauge gekocht. Aus der verseiften Masse ließ sich mit Äther kein Cholesterin ausziehen.

Die Acetonlösungen wurden nebeneinander eingeengt und auf $0^{\circ}$ abgekühlt, die abgeschiedenen Kristalle abfiltriert, die Mutterlauge wieder konzentriert, abgekühlt, die ausgeschiedenen Kristalle abermals abfiltriert usw., bis keine weitere Kristallisation mehr erfolgte. Die Hauptmenge des Cholesterins schied sich aus der ersten Acetonlösung ab. Aus der dritten wurde nur sehr wenig erhalten, aus der vierten gar nichts.

Die letzten Mutterlaugen, aus denen sich keine Kristalle mehr gewinnen ließen, wurden vereinigt. Sie stellten eine geringe Menge einer wasserhaltigen, braun gefärbten Flüssigkeit dar, welche sich mit Alkohol klar mischte. Nach der Verseifung mit alkoholischer Kalilauge ließ sich aus diesen Mutterlaugen kein Cholesterin isolieren.

Damit ist der Beweis erbracht, daß weder in dem in Aceton unlöslichen Teil des Ätherauszuges der Gehirne, noch in der nach Abscheidung des Cholesterins aus der Acetonlösung hinterbleibenden Mutterlauge eine Substanz, welche nach der Verseifung Cholesterin gibt, also gebundenes Cholesterin im Sinne von Baumstark, vorhanden ist.

Baumstark hat die Vermutung ausgesprochen, daß das von ihm als gebunden angenommene Cholesterin in Form des Ölsäureesters vorkäme, da er aus der verseiften Masse außer Cholesterin bedeutende Mengen Ölsäure zu isolieren vermochte.

Dort wo aber Baumstark das gebundene Cholesterin nachgewiesen $\mathrm{zu}$ haben glaubte, $d . \mathrm{h}$. in dem in Alkohol leicht löslichen Teil des Ätherauszuges, ist das Vorkommen von Cholesterinestern höchst unwahrscheinlich; denn nach den Untersuchungen von E. Schulze ${ }^{1}$ ) und den späteren von Hürthle ${ }^{2}$ ) sind die Cholesterinester in Alkohol schwer löslich. Baumstark selbst weist auf diesen Widerspruch hin.

Die bisher geschilderten Ergebnisse meiner Versuche sprechen nun keineswegs gegen die Existenz von Cholesterin-

1) Ber. d. deutsch. chem. Ges., 1872, S. 1075.

2) Diese Zeitschrift, Bd. XXI, S. 335.

Hoppe-Seyler's Zeitschrift f. physiol. Chemie. XLVI. 
estern im Gehirn, denn diese Substanzen werden aus ätherischer Lösung, in der sie neben Cholesterin vorhanden sind, ebensowenig wie dieses selbst durch Aceton ausgefällt, sie mußten daher, falls sie im Gehirn vorhanden waren, sich in den Acetonlösungen finden und mit dem Cholesterin zusammen beim Verdunsten der Lösungen auskristallisieren. Daß tatsächlich durch Aceton in ätherischer Lösung von Gemengen des Cholesterins mit seinen Estern keine Fällung hervorgerufen wird, konnte ich feststellen, indem ich ätherische Lösungen von Cholesterin, welches mit verschiedenen Mengen von Ölsäureester, Palmitinsäureester oder Stearinsäureester ${ }^{1}$ ) gemischt war, mit dem mehrfachen Volumen Aceton versetzte: in keinem Falle trat eine Abscheidung auf. Alle Lösungen blieben dauernd klar.

Es waren daher nur noch die aus den Acetonlösungen erhaltenen Cholesterinkristalle auf einen etwaigen Gehalt an Estern zu untersuchen. Nun sind einerseits die Cholesterinester sehr viel schwerer löslich in Alkohol als reines Cholesterin, andrerseits weicht ihr Schmelzpunkt ganz bedeutend (um 60 von dem des reinen Cholesterins $a b$, sodaß $\mathrm{zu}$ erwarten war, daß sich die Anwesenheit auch geringer Mengen von Estern neben Cholesterin dadurch zu erkennen geben würde, daß die aus einer warmen alkoholischen Lösung, welche beide Substanzen enthielt, zuerst auskristallisierende Fraktion einen niedrigeren Schmelzpunkt zeigt, als reines Cholesterin.

Daraufhin angestellte Versuche mit Mischungen von Cholesterin mit wechselnden Mengen verschiedener Ester ergaben, da $B$ in der Tat geringe Beimengungen von Estern den Schmelzpunkt der beim Erkalten einer warmen alkoholischen Lösung zuerst auftretenden Kristalle herunterdrückte, während die aus der eingeengten Mutterlauge erhaltenen Kristalle sich dem richtigen Schmelzpunkt des Cholesterins näherten. Umstehende Tabelle gibt eine Übersicht über die bei diesen Versuchen gefundenen Schmelzpunkte:

1) Die Ester waren nach den Angaben von Hürthle (l. c.) synthetisch dargestellt. Sie stimmten in allen Eigenschaften mit den für diese Substanzen angegebenen überein und zeigten die richtigen Schmelzpunkte: Der Ölsäureester schmolz bei $42^{\circ}$, der Palmitinsäureester bei $78^{\circ}$ und der Stearinsäureester bei $82^{\circ}$. 


\begin{tabular}{c|c|c|c}
\hline \hline $\begin{array}{c}\text { Alkoholische Cholesterin- } \\
\text { lösung } \\
\text { mit }\end{array}$ & $\begin{array}{c}\mid 3 \\
\text { zuerst } \\
\text { erhaltenen } \\
\text { Kristalle }\end{array}$ & $\begin{array}{c}\text { aus der ersten } \\
\text { Mutterlauge erhaltenen Kristalle }\end{array}$ & aus der zweiten \\
\hline $5 \%$ Ölsäureester . . & $80^{\circ}$ & $142^{\circ}$ & $145^{\circ}$ \\
$5 \%$ Palmitinsäurester . & $80^{\circ}$ & $141^{\circ}$ & $145^{\circ}$ \\
$5 \%$ Stearinsäureester . & $75^{\circ}$ & $140^{\circ}$ & - \\
$10^{\circ} \%$ Ölsäureester . . & $36^{\circ}$ & $120^{\circ}$ & $142^{\circ}$ \\
$10^{\circ} \%$ Palmitinsäureester . & $50^{\circ}$ & $139^{\circ}$ & - \\
$10^{\circ} \%$ Stearinsäureester & $60^{\circ}$ & $140^{\circ}$ & -
\end{tabular}

Nachdem also festgestellt worden war, daß auf diese Weise schon kleine Mengen von Cholesterinestern neben Cholesterin nachgewiesen werden können, kristallisierte ich die Gesamtmenge der erhaltenen Kristalle (siehe oben) aus Alkohol um und bestimmte den Schmelzpunkt der zuerst sich abscheidenden Kristalle. Er lag bei $142^{\circ}$, um nach abermaligem Umkristallisieren auf $145^{\circ} \mathrm{zu}$ steigen. Danach ist also die Anwesenheit auch kleiner Mengen von Cholesterinestern im Gehirn auszuschließen.

Zur Kontrolle wurden sämtliche Cholesterinkristalle noch nach der von Kossel und Obermüller ${ }^{1}$ ) angegebenen Methode mit Natriumalkoholat behandelt. -Es konnten dabei keine Fettsäuren isoliert werden.

Das Ergebnis meiner Versuche ist, daß sich im Ätherauszug des Gehirns weder Ester des Cholesterins mit höheren Fettsäuren, noch andere Verbindungen des Cholesterins, welche beim Verseifen gespalten werden, finden.

1) Diese Zeitschrift, Bd. XVI, S. 141. 